SCIENTIFIC LETTER

\title{
Impact of pre-diabetic state on clinical outcomes in patients with acute coronary syndrome
}

\author{
R Otten, E Kline-Rogers, D J Meier, R Dumasia, J Fang, N May, Y Resin, D F Armstrong, F Saab, \\ $M$ Petrina, K A Eagle, D Mukherjee
}

D iabetes mellitus is a well recognised risk factor for cardiovascular disease and diabetic individuals with acute coronary syndrome (ACS) have a two- to fourfold increased risk of adverse cardiovascular events compared to non-diabetic individuals. ${ }^{1}$ It is becoming increasingly clear that impaired glucose metabolism and the pre-diabetic state are also associated with adverse clinical outcomes. A retrospective study of 197 patients by Norhammar et al ${ }^{2}$ showed that among non-diabetic patients with acute myocardial infarction, those with higher admission blood glucose had higher rates of death, rehospitalisation for heart failure, and rehospitalisation for non-fatal reinfarction. In this study, admission plasma glucose was an independent predictor of non-fatal reinfarction, hospitalisation for heart failure, and a major adverse cardiovascular event (MACE). ${ }^{2}$ Another study by Norhammar et al revealed that $35 \%$ of patients admitted to the coronary care unit with a myocardial infarction and no prior diagnosis of diabetes may have an abnormal glucose tolerance test at discharge. ${ }^{3}$

The American Diabetes Association (ADA) recently redefined the cut off point for normal fasting blood glucose concentrations from $110 \mathrm{mg} / \mathrm{dl}$ to $100 \mathrm{mg} / \mathrm{dl}$, meaning that a value of $100 \mathrm{mg} / \mathrm{dl}$ or above would lead to a diagnosis of impaired fasting glucose, which is included in the term prediabetes. ${ }^{4}$ The impact of the new guideline is immense. Health and human services estimate that $40 \%$ of US adults aged $40-74$ years, approximately 41 million people, have prediabetes. Under the previous criteria, it was estimated that 21 million people in this age group have pre-diabetes. We assessed the impact of the pre-diabetic state on clinical outcomes in patients presenting with ACS incorporating the new ADA definition.

\section{METHODS}

The primary objective was to analyse the prognostic implication of fasting glucose concentrations in ACS patients. We studied 1955 consecutive patients who were admitted to the University of Michigan Medical Center from January 1999 to August 2002 with a diagnosis of ACS. The protocol was approved by the institutional review board at the University of Michigan and informed consent was obtained from all patients.

All patients were initially identified by a discharge diagnosis of unstable angina or acute myocardial infarction. Identified charts were reviewed by nurses or physicians for entry criteria. Inclusion into the study required symptoms

Table 1 Demographics, management, and clinical outcomes of patients presenting with ACS stratified by fasting glucose concentrations

\begin{tabular}{|c|c|c|c|c|c|}
\hline & \multirow{2}{*}{$\begin{array}{l}\text { Fasting glucose } \\
<100 \mathrm{mg} / \mathrm{dl} \text { or } \\
5.6 \mathrm{mmol} / \mathrm{l} \\
\mathrm{n}=579\end{array}$} & \multirow{2}{*}{$\begin{array}{l}\text { Fasting glucose } 100-126 \\
\mathrm{mg} / \mathrm{dl} \text {, or } 5.6-7.0 \mathrm{mmol} / \mathrm{l} \\
\mathrm{n}=407\end{array}$} & \multirow{2}{*}{$\begin{array}{l}\text { Fasting glucose } 110-126 \\
\mathrm{mg} / \mathrm{dl} \text {, or } 6.1-7.0 \mathrm{mmol} / \mathrm{l} \\
\mathrm{n}=192\end{array}$} & \multirow{2}{*}{$\begin{array}{l}\text { Diabetic } \\
\mathrm{n}=777\end{array}$} & \multirow[b]{2}{*}{ p Value } \\
\hline & & & & & \\
\hline \multicolumn{6}{|l|}{ Presentation } \\
\hline ST elevation MI & $8.8 \%$ & $18.6 \%$ & $20.8 \%$ & $13.2 \%$ & $<0.001$ \\
\hline Non-ST elevation MI & $48.3 \%$ & $48.4 \%$ & $52.1 \%$ & $57.1 \%$ & 0.001 \\
\hline Unstable angina & $42.8 \%$ & $32.9 \%$ & $27.1 \%$ & $29.6 \%$ & $<0.001$ \\
\hline \multicolumn{6}{|l|}{ Demographics } \\
\hline Mean age (years) & $63.9(14.8)$ & $64.2(14.2)$ & $65.4(14.1)$ & $65.2(13.1)$ & 0.281 \\
\hline Female & $33.8 \%$ & $29.9 \%$ & $29.6 \%$ & $40.5 \%$ & 0.005 \\
\hline Body mass index $\left(\mathrm{kg} / \mathrm{m}^{2}\right)$ & $27.0(5.1)$ & $28.8(5.9)$ & $29.0(6.4)$ & $30.2(6.8)$ & $<0.001$ \\
\hline Prior MI & $45.1 \%$ & $38.3 \%$ & $39.5 \%$ & $48.6 \%$ & 0.006 \\
\hline Prior TIA/stroke & $10.9 \%$ & $9.1 \%$ & $11.4 \%$ & $14.1 \%$ & 0.064 \\
\hline Hypertension & $66.4 \%$ & $66.5 \%$ & $64.5 \%$ & $78.9 \%$ & $<0.001$ \\
\hline Hyperlipidaemia & $62.4 \%$ & $61.9 \%$ & $61.1 \%$ & $63.9 \%$ & 0.840 \\
\hline Renal insufficiency & $9.67 \%$ & $8.11 \%$ & $7.29 \%$ & $23.8 \%$ & $<0.001$ \\
\hline Peripheral vascular disease & $10.1 \%$ & $12.1 \%$ & $14.7 \%$ & $16.1 \%$ & 0.011 \\
\hline Positive biomarkers & $56.3 \%$ & $66.5 \%$ & $72.9 \%$ & $68.9 \%$ & $<0.001$ \\
\hline Ejection fraction (\%) & $55.6(14.6)$ & $53.5(15.3)$ & $52.2(15.5)$ & $48.1(17.3)$ & $<0.001$ \\
\hline Peak troponin & 15.7 (58.95) & $20.9(49.47)$ & 49.1 (107.3) & $38.4(104.5)$ & 0.182 \\
\hline \multicolumn{6}{|l|}{ Management } \\
\hline Coronary angiography & $65.8 \%$ & $71.2 \%$ & $70.3 \%$ & $62.5 \%$ & 0.027 \\
\hline $\mathrm{PCl}$ & $38.6 \%$ & $41.0 \%$ & $43.2 \%$ & $33.5 \%$ & 0.016 \\
\hline CABG & $7.6 \%$ & $11.1 \%$ & $11.4 \%$ & $10.2 \%$ & 0.200 \\
\hline IV GP Ilb/IIla & $23.4 \%$ & $30.2 \%$ & $31.7 \%$ & $21.4 \%$ & 0.001 \\
\hline Thrombolytics & $2.25 \%$ & $1.97 \%$ & $2.60 \%$ & $2.32 \%$ & 0.964 \\
\hline
\end{tabular}

CABG, coronary artery bypass surgery; IV GP Ilb/Illa, intravenous glycoprotein Ilb/Illa antagonist; Ml, myocardial infarction; PCl, percutaneous coronary intervention; TIA, transient ischaemic attack; $p$ values are for comparison across all four groups. 
Table 2 Clinical outcomes in patients with ACS stratified by fasting glucose concentrations

\begin{tabular}{|c|c|c|c|c|c|}
\hline & \multirow{2}{*}{$\begin{array}{l}\text { Fasting glucose }<100 \\
\mathrm{mg} / \mathrm{dl} \text { or } 5.6 \mathrm{mmol} / \mathrm{l} \\
\mathrm{n}=579\end{array}$} & \multirow{2}{*}{$\begin{array}{l}\text { Fasting glucose } 100-126 \\
\mathrm{mg} / \mathrm{dl} \text {, or } 5.6-7.0 \mathrm{mmol} / \mathrm{l} \\
\mathrm{n}=407\end{array}$} & \multirow{2}{*}{$\begin{array}{l}\text { Fasting glucose } 110-126 \\
\mathrm{mg} / \mathrm{dl} \text {, or } 6.1-7.0 \mathrm{mmol} / \mathrm{l} \\
\mathrm{n}=192\end{array}$} & \multirow{2}{*}{$\begin{array}{l}\begin{array}{l}\text { Diabetic } \\
\text { patients }\end{array} \\
n=777\end{array}$} & \multirow[b]{2}{*}{ p Value } \\
\hline & & & & & \\
\hline Death & $1.04 \%$ & $1.23 \%$ & $1.56 \%$ & $6.69 \%$ & $<0.001$ \\
\hline Reinfarction & $0.49 \%$ & $1.18 \%$ & $0 \%$ & $0.96 \%$ & 0.544 \\
\hline Stroke & $0.17 \%$ & $0 \%$ & $0 \%$ & $1.16 \%$ & 0.561 \\
\hline Cardiogenic shock & $0.52 \%$ & $1.47 \%$ & $2.60 \%$ & $7.86 \%$ & $<0.001$ \\
\hline Pulmonary oedema & $2.21 \%$ & $3.53 \%$ & $3.36 \%$ & $10.8 \%$ & $<0.001$ \\
\hline Cardiac arrest & $0.69 \%$ & $1.97 \%$ & $2.60 \%$ & $5.68 \%$ & $<0.001$ \\
\hline Atrial fibrillation/flutter & $4.8 \%$ & 7.15 & $10.4 \%$ & $8.5 \%$ & 0.022 \\
\hline MACE* & $6.74 \%$ & $12.04 \%$ & $15.63 \%$ & $20.21 \%$ & $<0.001$ \\
\hline \multicolumn{6}{|c|}{ Multivariate risk adjusted effect of fasting blood glucose concentrations on clinical outcome†‡ } \\
\hline \multirow{2}{*}{\multicolumn{2}{|c|}{ Fasting glucose $100-126 \mathrm{mg} / \mathrm{dl}$ or $5.6-7.0 \mathrm{mmol} / \mathrm{L}$}} & $1.66(1.05$ to 2.61$)$ & & $p=0.028$ & \\
\hline & & $2.10(1.19$ to 3.41$)$ & & $p=0.008$ & \\
\hline \multicolumn{2}{|c|}{ Diabetic patients } & 3.00 (2.05 to 4.39$)$ & & $\mathrm{p}<0.001$ & \\
\hline
\end{tabular}

*MACE includes death, reinfarction, stroke, cardiogenic shock, pulmonary oedema, cardiac arrest, and atrial dysrythmia; todds ratios and $95 \%$ confidence intervals; the $c$ index for the model was 0.726 , suggesting good model discrimination, and the Hosmer-Lemeshow test statistic was 0.45 , suggesting adequate model calibration and goodness of fit; $p$ values are for comparison across all four groups.

consistent with acute coronary insufficiency, along with one or more of the following: a history of coronary artery disease, electrocardiographic changes suggestive of ischaemia, evidence of coronary artery disease by catheterisation, and/or elevation of cardiac biomarkers.

Clinical, demographic, treatment, and outcome data were abstracted from medical charts by trained abstractors (physicians and/or cardiology research nurses). Demographic variables included age and sex. Co-morbidities included prior history of heart disease (angina, heart failure, myocardial infarction, coronary artery bypass grafting, and percutaneous coronary intervention), diabetes, smoking, hyperlipidaemia, and hypertension. ECG changes and initial laboratory data including fasting plasma glucose were recorded. Procedures and complications during the ACS hospitalisation were documented.

Patients were stratified based on their fasting glucose concentrations as non-diabetic, pre-diabetic based on both the new and the old ADA definitions, and those with known diabetes. We compared in-hospital outcomes including death, reinfarction, stroke, cardiogenic shock, pulmonary oedema, cardiac arrest, atrial dysrhythmias, and the composite of MACE in the different categories.

Univariate statistics are presented as frequency and percentage for categorical variables, and mean (SD) for continuous variables. $p$ Values for comparisons of the distributions of categorical variables between groups were based on $\chi^{2}$ tests. $p$ Values for comparisons of continuous variables between diabetic and non-diabetic groups were based on $t$ tests. A multivariable logistic regression analysis was performed for in-hospital MACE in ACS patients adjusted for age, sex, troponin elevation, ST segment elevation, serum creatinine, heart failure, and revascularisation. All analyses were performed using SAS 8.2 (SAS Institute, Cary, North Carolina, USA).

\section{RESULTS}

Patients with impaired fasting glucose or pre-diabetes were more likely to be male, have higher body mass index, have higher incidence of peripheral vascular disease, and a lower left ventricular ejection fraction (table 1). Adverse clinical events including pulmonary oedema, cardiogenic shock, and cardiac arrest were all significantly higher in pre-diabetic and diabetic patients compared to those with normal fasting glucose (table 2). Multivariate risk adjustment demonstrated a gradient of risk for adverse clinical outcomes in patients with pre-diabetes proportional to fasting glucose concentrations. Patients previously excluded from the definition of prediabetes-that is, those with fasting plasma glucose 100$110 \mathrm{mg} / \mathrm{dl}$ - had a $31 \%$ higher risk of MACE (odds ratio (OR) $1.31,95 \%$ confidence interval (CI) 0.73 to 2.35 ) compared to non-diabetic patients. Patients with the new ADA definition of pre-diabetes had a 66\% increased risk of MACE (OR 1.66, $95 \%$ CI 1.05 to 2.61$)$ compared to non-diabetic patients (table 2, fig 1).

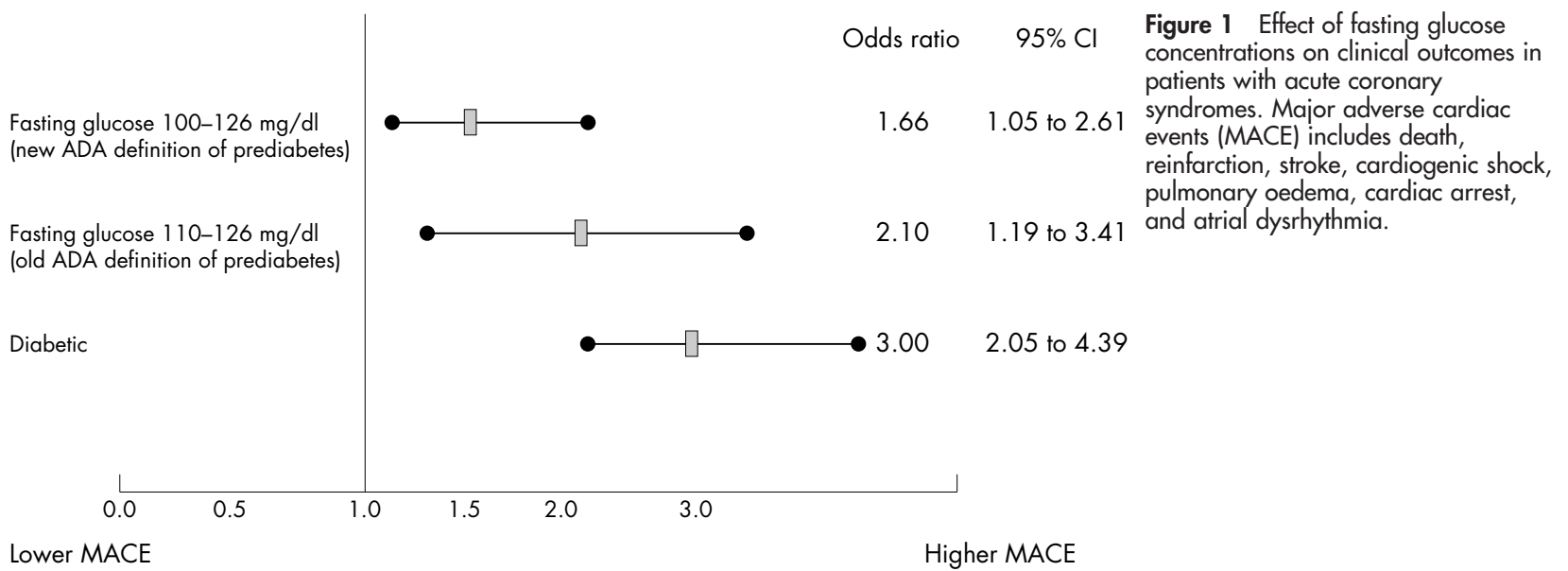




\section{DISCUSSION}

The goal of the ADA's new definition for pre-diabetes was to improve the sensitivity of identifying an at risk population for developing diabetes later in life. ${ }^{4}$ We demonstrate that the pre-diabetic state is also a marker for worse prognosis in patients with ACS. Our results are congruent with prior studies which demonstrated that patients with elevated fasting blood sugars not diagnostic of diabetes still have an increased long term risk of adverse cardiac events. ${ }^{5}$ The current study demonstrates that using the new ADA definition the population of the patients with pre-diabetes was doubled. Thus, the new ADA definition identifies more patients with impaired fasting glucose who may be targeted for optimal treatments to improve clinical outcomes. This study validates the higher risk profile in patients with impaired fasting glucose identified by the new ADA definition.

This study provides independent confirmation of the prognostic significance of a fasting glucose $\geqslant 100 \mathrm{mg} / \mathrm{dl}$ for patients presenting with ACS. Such patients would then be candidates for potential benefits from aggressive acute phase treatment (for example, early invasive strategy, glycoprotein IIb/IIIa receptor inhibitors) and long term use of optimal secondary prevention treatments, such as aspirin, clopidogrel, statins, $\beta$ blockers, and angiotensin converting enzyme inhibitors. It remains to be determined whether insulin sensitisers, such as metformin or a thiazolidinedione, might further improve clinical outcomes in pre-diabetic patients presenting with ACS. For now, in addition to evidence based pharmacotherapies, appropriate lifestyle modifications such as regular exercise and dietary modifications should be a major component of secondary prevention in these high risk pre-diabetic patients.

\section{ACKNOWLEDGEMENTS}

This study was supported in part by the Mardigian Foundation Grant.

\section{Authors' affiliations}

R Otten, E Kline-Rogers, D J Meier, R Dumasia, J Fang, N May, Y Resin, D F Armstrong, F Saab, M Petrina, K A Eagle, Department of Internal Medicine, University of Michigan, Ann Arbor, Michigan, USA

D Mukherjee, Gill Heart Institute, University of Kentucky, Lexington, Kentucky, USA

Correspondence to: Debabrata Mukheriee, MD, FACC, Gill Heart Institute, Division of Cardiovascular Medicine, 326 Wethington building, 900 S Limestone, Lexington, Kentucky 40536-0200, USA; mukherjee@ uky.edu

\section{REFERENCES}

1 Fergus TS, Fazel R, Fang J, et al. Presentation, management, and outcomes of diabetic patients compared to non-diabetic patients admitted for acute coronary syndromes. Heart 2004:90:1051-2.

2 Norhammar AM, Ryden L, Malmberg K. Admission plasma glucose. Independent risk factor for long-term prognosis after myocardial infarction even in nondiabetic patients. Diabetes Care 1999;22:1827-31.

3 Norhammar A, Tenerz A, Nilsson G, et al. Glucose metabolism in patients with acute myocardial infarction and no previous diagnosis of diabetes mellitus: a prospective study. Lancet 2002;359:2140-4.

4 Anon. Diagnosis and classification of diabetes mellitus. Diabetes Care 2004;27(suppl 1):S5-S10.

5 Biornholt JV, Erikssen G, Aaser E, et al. Fasting blood glucose: an underestimated risk factor for cardiovascular death. Results from a 22-year follow-up of healthy nondiabetic men. Diabetes Care 1999;22:45-9.

\section{IMAGES IN CARDIOLOGY}

\section{Acute dyspnoea two days after chest pain}

A 78 year old woman presented to a local hospital 10 hours after onset of typical angina. Based on symptoms of acute chest pain, ST elevation in anterior leads, and elevated creatine phosphokinase (maximum value $450 \mathrm{U} / \mathrm{l}$ ), the diagnosis of an acute anterior myocardial infarction (AMI) was made. Because of the circumstances a conservative management was pursued. Twenty four hours later, after initial recovery, the patient presented with acute dyspnoea caused by pulmonary oedema and had to be intubated. For further diagnostics and treatment she was transferred to our institution. Transthoracic and transoesophageal ultrasound showed a ruptured posterior papillary muscle (panel A). Coronary angiography revealed a mild three vessel disease with a relevant stenosis of the left anterior descending artery (LAD). Due to low output an intra-aortic balloon pump was inserted. Emergency mitral valve replacement (MVR) combined with revascularisation of the LAD was performed (panel B). The surgical procedure was well tolerated. Further recovery was complicated by progressive respiratory distress syndrome with pneumonia leading to death of the patient 10 days later.

This case illustrates the typical occurrence of a mechanical complication after a relatively small AMI. Mechanical complications following AMI occurs relatively rarely

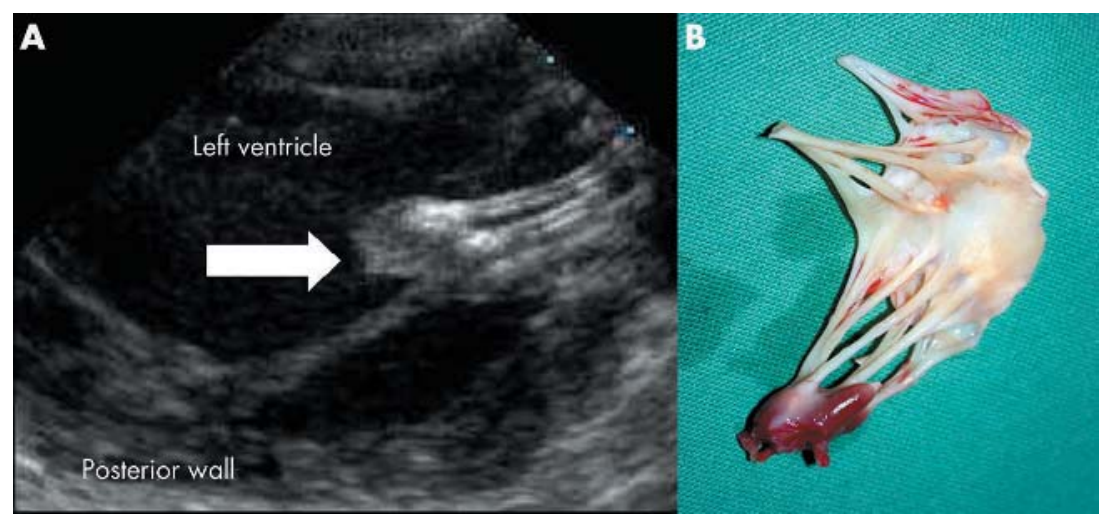

Panel A: Transoesophageal long axis view. Ruptured papillary muscle (arrow from the left). Panel B: Surgically resected mitral valve with necrotic ruptured papillary muscle.

$(2-3 \%)$, but need to be diagnosed and treated as quickly as possible. Mechanical complications should be considered in any patient deteriorating acutely after a preceding ischaemic myocardial event and initial stabilisation. Once the diagnosis of an ischaemic papillary muscle rupture is made, immediate surgical intervention should be considered and undertaken. 